OPEN

SUBJECT AREAS:

CELL LINEAGE

ORGANOGENESIS

Received

27 August 2014

Accepted

15 October 2014

Published

5 November 2014

Correspondence and requests for materials should be addressed to M.J.C. (mjcohn@ufl. edu)

\title{
Embryonic origin and compartmental organization of the external genitalia
}

\author{
Ana M. Herrera' \& Martin J. Cohn ${ }^{1,2}$
}

'Department of Molecular Genetics and Microbiology, Howard Hughes Medical Institute, UF Genetics Institute, University of Florida, P.O. Box 103610, Gainesville, FL 32610, USA, ${ }^{2}$ Department of Biology, Howard Hughes Medical Institute, UF Genetics Institute, University of Florida, P.O. Box 103610, Gainesville, FL 32610, USA.

Genital malformations occur at a high frequency in humans, affecting $\sim 1: 250$ live births. The molecular mechanisms of external genital development are beginning to be identified; however, the origin of cells that give rise to external genitalia is unknown. Here we use cell lineage analysis to show that the genital tubercle, the precursor of the penis and clitoris, arises from two populations of progenitor cells that originate at the lateral edges of the embryo, at the level of the posterior hindlimb buds and anterior tail. During body wall closure, the left and right external genital progenitor pools are brought together at the ventral midline, where they form the paired genital swellings that give rise to the genital tubercle. Unexpectedly, the left and right external genital progenitor pools form two lineage-restricted compartments in the phallus. Together with previous lineage studies of limb buds, our results indicate that, at the pelvic level, the early lateral mesoderm is regionalized from medial to lateral into dorsal limb, ventral limb, and external genital progenitor fields. These findings have implications for the evolutionary diversification of external genitalia and for the association between external genital defects and disruption of body wall closure, as seen in the epispadias-extrophy complex.

\footnotetext{
Q ongenital defects of the external genitalia occur at a remarkable frequency in humans, with hypospadias (incomplete closure of the urethral tube) affecting $\sim 1: 250$ live births ${ }^{1-3}$. Over the past decade, our understanding of the molecular genetics of external genital development has improved, and the mechanisms of genital outgrowth and urethral tube closure are beginning to be understood. Most of the progress in the area of external genital development has come from work on the mouse model; however, recent comparative studies have shown that early development of external genitalia is evolutionarily conserved across most amniote vertebrates ${ }^{4}$. In mammals, birds, and each of the major reptile clades, external genital development begins with the emergence of paired genital swellings on either side of the cloacal membrane ${ }^{5-11}$. The paired genital swellings then merge beneath the surface ectoderm to form a single genital tubercle anterior to the cloaca ${ }^{11}$ (although in squamates they remain unfused, forming 2 phalluses known as hemipenes or hemiclitores ${ }^{5,9}$ ). The genital tubercle is the precursor of the penis and the clitoris, although early development of the tubercle is similar in males and females. Sexual differentiation of the external genitalia occurs relatively late, after differentiation of the gonads and expression of sex steroid receptors in the genital tubercle. All three germ layers participate in external genital development; genital mesoderm forms the stromal tissue of the phallus, endodermally derived urethral plate epithelium forms the entire urethral tube, and a jacket of ectodermal epithelium forms the skin and epithelial appendages, such as spines and hair ${ }^{12,13}$. Although cell lineage analysis of different germ layers within the genital tubercle has identified the fates of genital tubercle derivatives, little is known about the embryonic origin of the genital tubercle itself.

Here we investigate the embryonic origin of external genitalia by mapping cell lineage of posterior lateral plate mesoderm and tail bud of chick embryos. We identified a population of cells at the lateral edge of the lateral mesoderm, at the level of the posterior hindlimb bud and anterior tail bud, that gives rise to the genital tubercle (Fig. 1a). This population exists on the left and right sides of the embryo, lateral to the previously identified hindlimb field (in chicks, the hindlimbs form opposite somites 26-33; ref. 14). Closure of the body wall brings the left and right genital progenitor fields together at the ventral midline, where they form the paired genital swellings and genital tubercle. Below we report on the origin of the external genitalia and the discovery of previously unknown cell lineage restricted compartments in the phallus.
} 

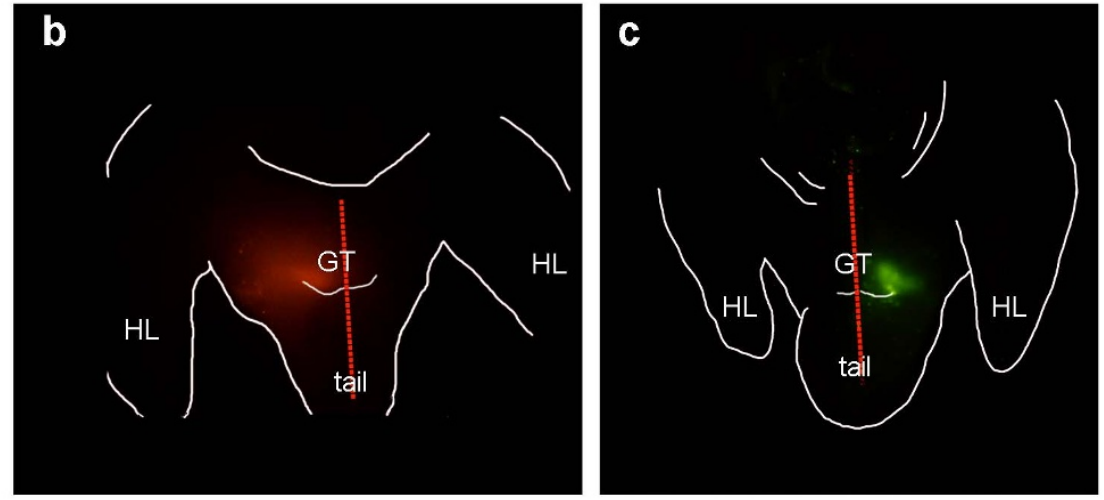

Figure 1 Identification of external genital progenitors in posterior lateral plate/anterior tail mesoderm of chick embryos. (a) Schematic diagram showing a dorsal view of a stage 18 chick embryo. Colored dots show injection sites for embryos shown in panels b (DiI, red dot) and c (DiA, green dot). (b) Ventral view of a chick embryo 48 hours after injection of DiI into the somatic layer of the lateral plate mesoderm, lateral to the posterior region of the right hindlimb (depicted by the red dot in panel a). DiI labeled cells are seen on right side of genital tubercle (left in ventral view). (c) Ventral view of a chick embryo 48 hours after injection of DiA into the somatic layer of the lateral plate mesoderm, lateral to the posterior region of the left hindlimb bud (depicted by the green dot in panel a). DiA labeled cells are visible on left side of genital tubercle (right in ventral view). GT, Genital tubercle; HL, hindlimb. Red broken line marks the position of the urethral plate epithelium.

\section{Results}

The genital tubercle is derived from the lateralmost mesoderm. Based on the anteroposterior position of the genital tubercle relative to the hindlimb buds, we postulated that progenitors of the paired genital swellings and the resultant genital tubercle could reside in lateral mesoderm at the axial level of the hindlimb buds. In order to test this hypothesis and to determine the origin of external genital progenitor cells, the lipophilic dyes DiI and DiA were used to label lateral mesodermal cells along the anteroposterior axis of chick embryos at stage 18, when hindlimb budding is initiated and before the emergence of the paired genital swellings. The distribution of labeled cells was examined after 48 hours of incubation. Because injections into the coelom resulted in dispersal of dye throughout the coelomic cavity and, consequently, nonspecific labeling of cells, the depth of dye injection was controlled so only cells in the somatic layer of the lateral plate mesoderm, superficial to the coelomic cavity, were labeled. When DiI and DiA injections were targeted to the lateral edges of the lateral plate mesoderm, between the posterior $1 / 5$ of the hindlimb bud and anterior region of the tail bud, labeled cells were found to contribute to the genital tubercle and cloaca (Fig. 1). Interestingly, cells labeled on the right and left sides of the embryo contributed only to the right and left sides of the tubercle, respectively (Fig. 1b, c). In no cases were labeled cells observed on the contralateral side of the genital tubercle.

To resolve the anterior and posterior limits of the external genital field, we performed fine-scale fate mapping of lateral mesodermal cells at discrete anteroposterior levels, from the hindlimb bud to the posterior part of the tail. The lateralmost region of the lateral plate and tail mesoderm was subdivided into four zones at the following axial levels; the posterior $1 / 5$ of the hindlimb bud (zone 2), the anterior part of the tail immediately posterior to the hindlimb budtail junction (zone 3), the posterior part of the tail bud (zone 4), and the region of the hindlimb anterior to zone 2 (zone 1; Fig. 2a).

Single color labeling was done by injecting dye into a single zone on one side of the embryo and double-labeling was done by injecting different color dyes either in the same zone on opposite sides of the embryo (e.g., zone 2 on the left and right sides; Fig. 2a, b) or in two zones on the same side (e.g., zones 2 and 3 on the right side only; Fig. 2f). Injections targeted to zone 2 resulted in labeling of the genital tubercle in 27 of 30 embryos (Fig. 2a-c; Fig. 3). Some injections into zone 2 labeled the genital tubercle and posterior cloacal swelling, which will form the posterior cloacal lip (Fig. 2b). In cases where zone 3 was injected, labeled cells were identified in the ventral part of the genital tubercle and/or anterior part of the tail (Fig. 2d, e). For example, 3 embryos showed labeling of the genital tubercle and anterior tail (Fig. 2d), and the remaining embryos showed labeled cells in the anterior tail only (Fig. 2e). In some cases, the injections labeled a larger population of cells over multiple zones, and this resulted in a wider range of cellular contributions, including the cloacal swellings anterior and posterior to the genital tubercle (Fig. 2c). When lateral plate mesoderm cells in zone 1 were injected, labeled cells contributed to the body wall immediately anterior to the genital tubercle, but not to the genital tubercle itself ( $n=7$; Fig. 3). Finally, we targeted cells in the posterior half of the tail bud (zone 4) and in all cases labeled cells contributed exclusively to the tail (Fig. 2g, $\mathrm{h}$ and Fig. 3).

When embryos were double labeled at two positions in zones 1-4, cells remained in register to the sites of injection (i.e., there was little or no intermingling of cells labeled at different anteroposterior levels), which resulted in separate clusters of clones along the anteroposterior axis of the body wall (see Fig. $2 \mathrm{f}-\mathrm{h}$ for examples). Cells labeled in zones 2 and 3 maintained their relative anterior-posterior positions, with cells from zone 2 contributing to the genital tubercle and cells from zone 3 contributing to the ventral genital tubercle and/ or anterior tail (Fig. 2f). When cells in zones 2 and 4 were labeled, zone 2 descendants were identified in the genital tubercle but zone 4 descendants contributed only to the tail (Fig. 2 g, h). Thus, $90 \%$ of the injections targeted to zone 2 (adjacent to the posterior region of the prospective the hindlimb bud) resulted in labeling of the genital tubercle, and half of the injections targeted to zone 3 (anterior tail) contributed to the genital tubercle, with the remaining injections in this region labeling cells in the anterior part of the tail (Fig. 3). Taken together, these results show that the genital tubercle originates from a zone of the lateralmost mesoderm that extends from the level of the posterior hindlimb bud to the anterior region of the tail bud. We refer to this domain of genital tubercle progenitor cells as "the external genital field", indicated by purple in the schematic diagram shown in Fig. 3.

The external genital field is adjacent to the hindlimb field in lateral plate mesoderm. Injections were targeted to the center of each zone in the above experiments; however, when the injection position was varied within a zone, the spatial relationship of the external genital field to the hindlimb progenitor field was revealed. For example, when DiI was injected in the anterior and medial side of 
a
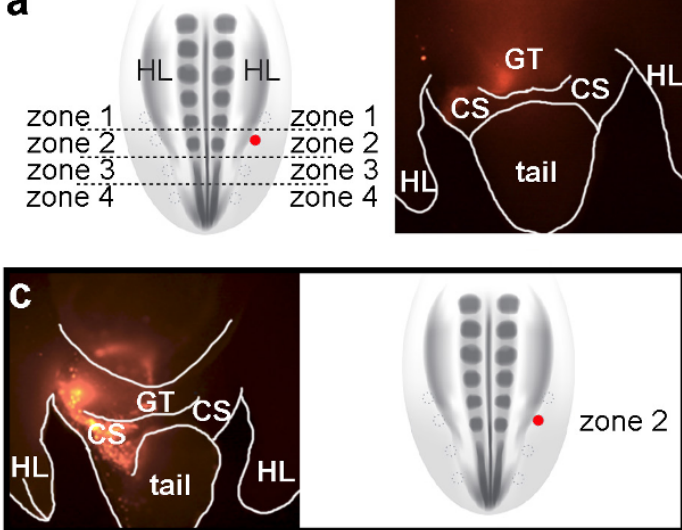

$\mathbf{e}$
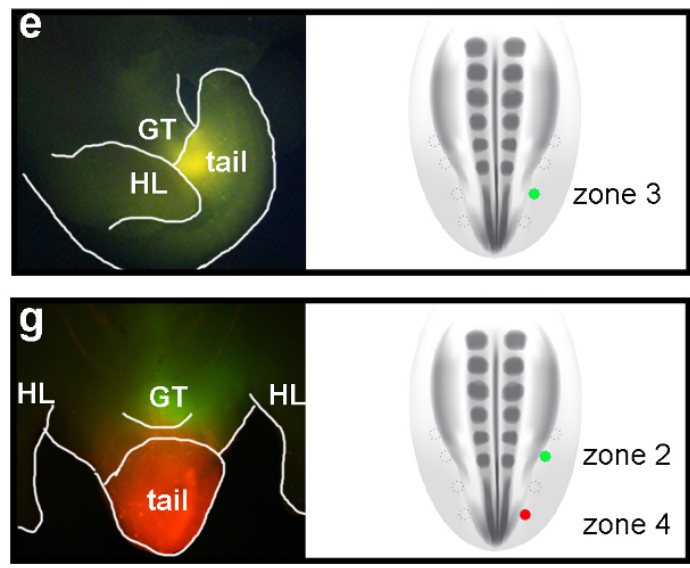
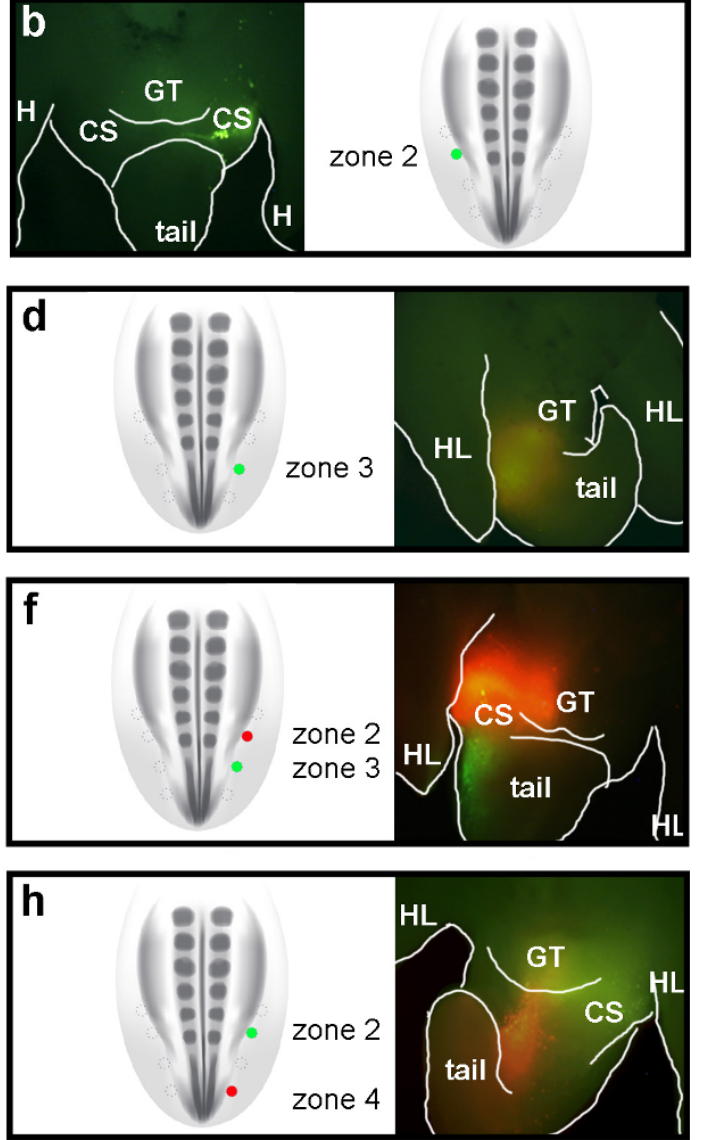

Figure $2 \mid$ Defining the anteroposterior limits of the external genital progenitor field in the chick lateral mesoderm. Posterior lateral mesoderm was divided into 4 zones and cells were labeled with DiI or DiA to trace their lineage. In each panel, the schematic diagram is a dorsal view showing the position of dye injection (filled circles) relative to the other zones (empty circles). The adjacent fluorescent micrographs are ventral views showing the distribution of labeled cells 48 hours after injection. (a) Cells labeled with DiI in zone 2 on the right side of the embryo contribute to the right side of the genital tubercle (GT) and the right posterior cloacal swelling (CS). Note that right side of embryo is to the left in ventral views. (b) Cells labeled with DiA in zone 2 on the left side of the embryo contribute to the left side of the GT and the left posterior cloacal swelling. (c) Embryo labeled with DiI on the right side in zone 2. Labeled cells contributed to the right sides of the GT, anterior cloacal swelling, and anterior tail. (d, e) Embryos labeled on the right side with DiA in zone 3. After 48 hours, labeled cells contributed to the posterior part of the GT and the anterior region of the tail (d), or to the anterior region of the tail only (e). (f) Embryo double-labeled on the right side with DiI in zone 2 and DiA in zone 3. After 48 hours, DiI-labeled cells from zone 2 contribute to the right side of the GT and DiA-labeled cells from zone 3 are found posterior to the GT, on the right sides of the posterior cloacal swelling and tail. (g, h) Embryos doublelabeled on the right side with DiA in zone 2 and DiI in zone 4 . After 48 hours, DiA-labeled cells contribute to the GT and DiI-labeled cells are found in the tail.

zone 2, a contiguous population of labeled cells extended from the genital tubercle into the ventral-posterior side of the hindlimb bud on the same side (Fig. $4 \mathrm{a}-\mathrm{c}$ ). This contrasted with injections that were made further laterally and/or posteriorly in zone 2, when cells contributed to the genital tubercle but not to the limb bud (compare Fig. $4 \mathrm{~b}$ and $\mathrm{c}$ to $2 \mathrm{a}-\mathrm{c}$ ). These results indicate that within the lateral plate mesoderm, the progenitor cells of the genital tubercle are positioned adjacent to, and may partially overlap with, the progenitor pool that gives rise to the ventral side of the hindlimb bud $^{15-17}$.

The genital tubercle consists of two lineage-restricted compartments derived from the left and right sides of the embryo. In the lineage labeling experiments described above, we observed that labeled cells remained on the same side of the genital tubercle as the site of injection; lateral mesodermal cells labeled on the right side of the embryo gave rise to the right side of the genital tubercle and cells labeled on the left side of the embryo contributed to the left side of the genital tubercle. We therefore tested the hypothesis that the genital tubercle consists of two lineage-restricted compartments derived from the left and right lateral mesoderm, with the urethral epithelium acting as a compartment boundary. Cells in zone 2 were labeled with DiI on the right side and DiA on the left side (Fig. 5a). Forty-eight hours after injection (stage 25-26), double-labeled embryos were fixed, imaged in whole mount, and then sectioned in the transverse plane to map the positions of daughter cells. At the level of the genital tubercle, there was a strict left-right restriction of cells; DiI and DiA labeled cells contributed to only one side of the tubercle, and this always reflected the side of the original injection (Fig. 5b). Sections revealed that DiI and DiA labeled descendent cells expand throughout the right and left sides, respectively, up to the urethral plate epithelium, but cells rarely crossed the urethral plate to the contralateral side of the tubercle (Fig. $5 c$ and d). These results suggest that the genital tubercle consists of two lineage-restricted compartments, in which the left and right sides of the genital tubercle are composed of clones derived from the left and right external genital fields, respectively, and these two populations of progenitor cells abut one another at the urethral plate in the midline of the genital tubercle.

\section{Discussion}

An external genital field in posterior lateral plate mesoderm and anterior tail. In the fate mapping experiments reported above, the 

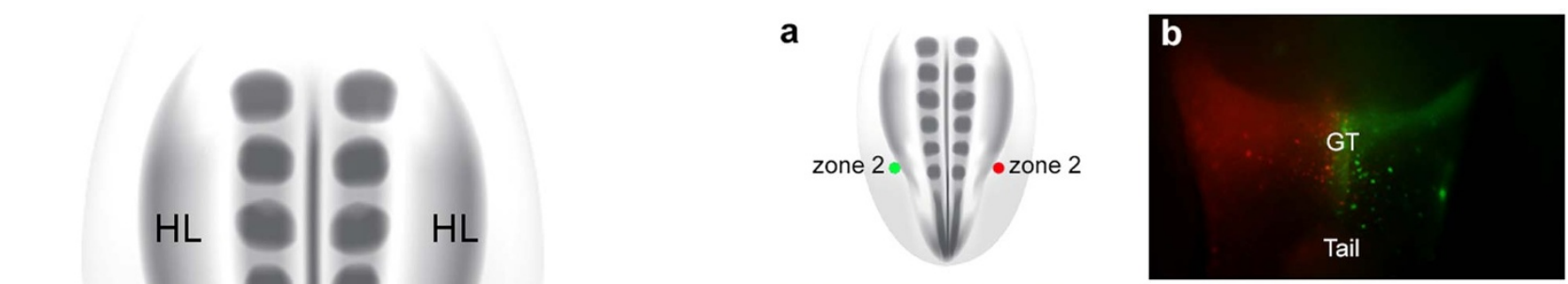

zone 1

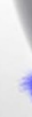

zone 2

zone 3

zone 4 zone 1

zone 2

zone 3

zone 4

Tail

\begin{tabular}{|c|c|c|}
\hline Site & Injections & GT contribution \\
\hline Zone 1 & 7 & 0 \\
\hline Zone 2 & 30 & 27 \\
\hline Zone 3 & 6 & 3 \\
\hline Zone 4 & 3 & 0 \\
\hline
\end{tabular}

Figure 3 The external genital field maps to the lateralmost mesoderm from the level of the posterior hindlimb bud to the anterior tail bud. Schematic diagram at top shows the distribution of the external genital progenitor cells (purple) in the chick embryo, dorsal view. The genital tubercle arises from cells situated in zone 2 (posterior hindlimb bud level) and the anterior region of zone 3 (anterior tail bud level). Table shows the results of DiI and DiA injections in each of the 4 zones and their contribution to the genital tubercle $(n=46)$. Ninety percent of the injections made in zone 2 labeled cells that contribute to the external genitalia. Fifty percent of the injections made in zone 3 labeled cells that contribute to the external genitalia. By contrast, cells labeled in zones 1 and 4 never contributed to the genital tubercle.

lateral mesodermal cells between the posterior region of the hindlimb bud and the anterior region of the tail give rise to the genital tubercle, demonstrating the existence of a field of external genital progenitor cells (zones 2 and 3 in Fig. 3). It is noteworthy that cells labeled at the anterior limit of zone 2 but slightly medial to the edge of the lateral plate contributed both to the genital tubercle and to the ventral side of the hindlimb bud, suggesting that the external genital field is lateral to, and may partially overlap with, with the hindlimb field. These findings are generally consistent with those obtained in a parallel study by Tschopp et $a l^{18}$. Previous fate mapping studies of the limb buds showed that the ventral limb bud progenitors are
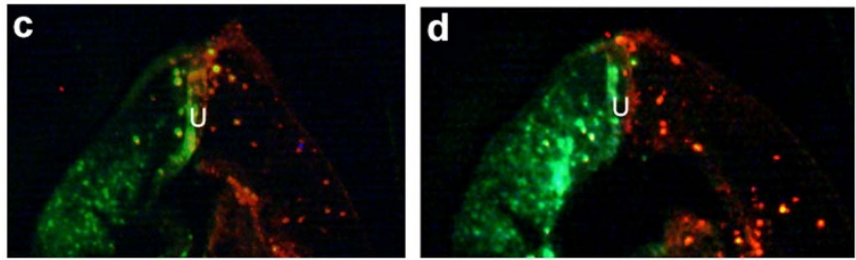

Figure $5 \mid$ The genital tubercle consists of two lineage-restricted compartments derived from left and right lateral mesoderm. (a) Schematic diagram of a dorsal view of a stage 18 chick embryo showing the position of two injections in zone 2, one on the right side (DiI, red dot) and one on the left side (DiA, green dot) of the lateralmost lateral plate mesoderm. (b) Ventral view 48 hours after injections. DiI-labeled cells are restricted to the right side (left in ventral view) and DiA-labeled cells are restricted to the left side (right in ventral view) of the genital tubercle. Cells originating from the left and right sides show little to no mixing at the midline of the genital tubercle. (c, d) Transverse sections through the genital tubercle of embryo shown in (b). Descendants of left and right progenitor pools remain restricted to the left and right sides of the genital tubercle. The boundary between the left and right compartments is the urethral plate $(\mathrm{U})$, and neither population crosses into the opposite mesenchymal compartment.

derived from the lateral side of the lateral plate, and the dorsal limb bud progenitors are derived from cells at more medial positions ${ }^{15-17}$. Our results indicate that regionalization along the mediolateral axis of the lateral plate extends to a third population of cells, the external genital progenitor cells, which are situated lateral to the ventral hindlimb progenitors (Fig. 6a). During body wall closure, these lateralmost cells are brought to the ventral midline, where they give rise to the paired genital swellings and, later, the genital tubercle (Fig. 6b).

Compartmental organization of the genital tubercle. Double labeling of left and right lateral plate mesoderm at hindlimb levels revealed that the genital tubercle develops from two progenitor pools that originate on either side of the embryo. As the body wall closes, the left and right populations of lateral plate mesoderm are brought together, merging at the ventral midline. The paired genital swellings that emerge on either side of the cloacal membrane are derived from the left and right genital progenitor pools, and these remain a

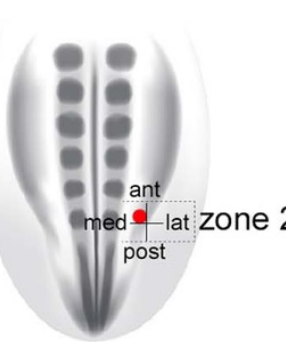

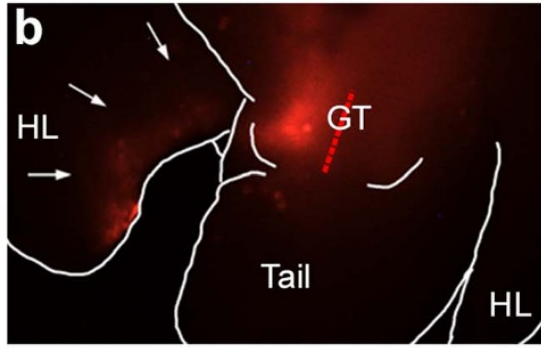

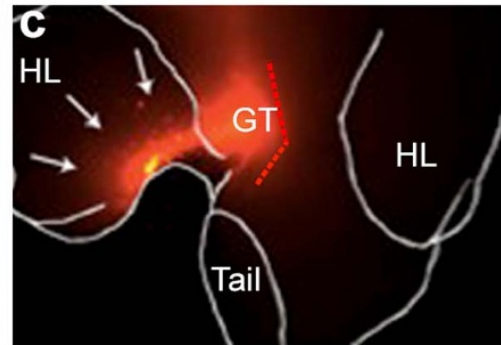

Figure 4 The external genital and ventral limb progenitor pools are contiguous (a) Schematic diagram of a dorsal view of a stage 18 chick embryo showing the position of a single injection of DiI (red dot) to the anterior-medial side of zone 2. (b, c) After 48 hours, labeled cells are restricted to the right side of the genital tubercle and the posterior aspect of the ventral side of the right hindlimb. Note labeled cells do not cross the midline of the genital tubercle (broken red line). 
a

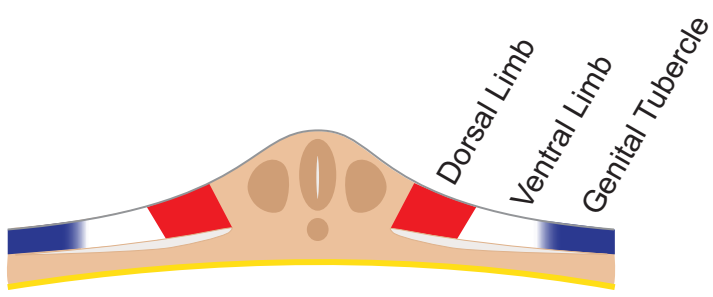

b

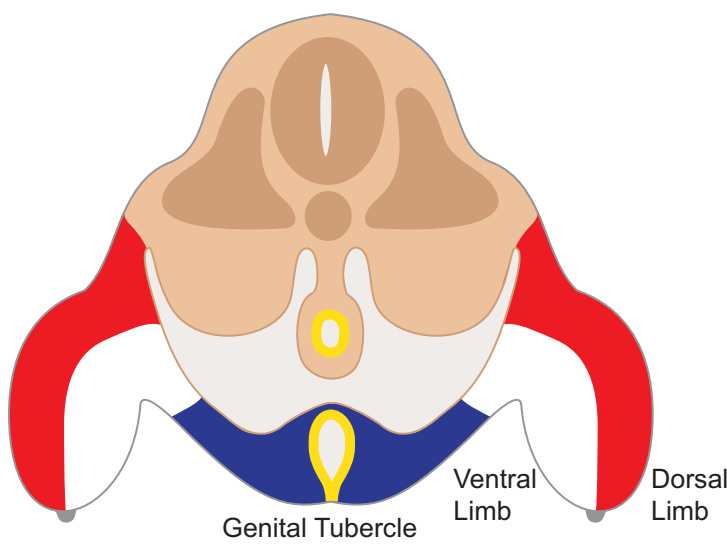

Figure 6 | Regionalization of lateral mesoderm into dorsal limb, ventral limb, and external genital fields along the mediolateral axis. Schematic diagram of transverse sections through the cloacal level of the embryo before (a) and after (b) closure of the body wall. (a) External genital progenitor cells (blue) originate at the lateral edges of the lateral mesoderm, adjacent to the ventral limb field (white). The dorsal limb field is shown in red. (b) Closure of the body wall brings together the left and right external genital fields at the ventral midline, where they give rise to the paired genital swellings that form the genital tubercle (blue). Limb buds are shown on the left and right sides, with the ventral limb shown in white and the dorsal limb shown in red.

separated by the cloacal endoderm that forms the urethral plate epithelium. Unexpectedly, after the paired genital swellings merge to form a single genital tubercle, cells derived from the left and right sides of the lateral plate mesoderm remain restricted to their respective sides of origin, and little mixing occurs at the midline. Based on these observations, we suggest that the genital tubercle exhibits true compartmental organization, sensu Drosophila compartments, in which adjacent cell populations show lineagerestriction and respect a compartment boundary ${ }^{19}$ (in this case, the urethral plate epithelium). Taken together with our recent findings that the origin of a genital tubercle from paired genital swellings is a shared feature of amniotes ${ }^{4-10}$, these results raise the possibility that left-right compartmental organization of the genital tubercle might be a broadly conserved character of amniote external genitalia.

Regionalization of lateral mesoderm: positioning of hindlimbs and genitalia. Paired genital swellings of amniotes vary in their anteroposterior and mediolateral positions (reviewed in ref. 4). In birds and mammals, for example, the paired genital swellings emerge anterior to the cloaca, whereas in squamates (lizards and snakes), the paired swellings arise posterior and lateral to the cloaca ${ }^{5,9}$. A striking shift is seen in the green lizard Anolis carolinensis, which undergoes initiation of paired genital swellings at the ventral-proximal end of the leg buds. In the context of the results presented here, this variation suggests that the boundary between the limb and genital fields in lateral plate mesoderm might be labile, and mediolateral shifting of this boundary could account for the variation in the positions of the genital swellings relative to the hindlimbs and cloaca. Our finding that cells labeled on the medial side of zone 2 were later found in the genital tubercle and on the ventral side of the posterior hindlimb bud suggests that the hindlimb and external genital fields might partially overlap or have a soft rather than a sharp boundary (although it is possible that these injections labeled cells on both sides of the boundary). Whether the external genital and hindlimb fields abut or partially overlap, the results presented here and in fate maps of the limb progenitors ${ }^{15-17}$ demonstrate that hindlimb and external genital progenitor pools are generally separate populations of cells. Variation in the relative positions of these appendages could result from shifts in the positions of the progenitor fields, although we cannot exclude the possibility that the limb and genital fields are generally fixed and it is the positions of outgrowth cues relative to these fields that are labile.

In squamates, the paired genital swellings remain separate throughout development, giving rise to the left and right hemipenes and hemiclitores ${ }^{4,5,9}$. We have not traced the lineage of squamate hemiphalluses; however, our results suggest that each hemiphallus could arise entirely from progenitor cells on one side of the embryo. Given that single median phalluses of mammals ${ }^{11}$, archosaurs ${ }^{7,8,10}$, and turtles ${ }^{6}$ develop from the fusion of left and right genital swellings, our cell lineage results might lend support to the idea that squamate external genital structures are true "hemi" (half) phalluses. This interpretation is not intended to suggest structural homology of the left and right hemipenis to the left and right side of the median phalluses of non-squamate amniotes, but rather to highlight the relationship between the left and right genital fields in the lateral plate mesoderm and the variation in amniote phallus morphology.

Our observation that cells labeled in zones 2 and 3 contribute to the dorsal (anterior relative to the trunk) and ventral (posterior) sides of the genital tubercle, respectively, suggests that external genital progenitor cells retain their original anteroposterior positions. These patterns of cell behavior are reminiscent of the anteroposterior organization of other structures, such as the chick scapular blade, in which cells retain the same relative positions as the somites from which they originate ${ }^{20}$. Based on our finding that genital tubercle cells are derived from the posteriormost lateral mesoderm in the trunk, adjacent to posterior hindlimb bud cells, it is tempting to speculate that positional identity of external genital progenitors, like limb progenitors, is specified during anteroposterior regionalization of the main body axis. Indeed, AbdB-related Hox genes are expressed at the posterior end of the embryo, in the genital tubercle, and in the posterior regions of the limb buds, and it is possible that the shared regulation of these genes in limbs and genitalia ${ }^{21}$ reflects their embryonic origin from the same axial level of the trunk.

Left-right compartmentalization of the genitalia and the relationship to epispadias-extrophy complex. Body wall closure during embryogenesis is critical for the enclosure of the internal organs, internalization of the gut, and formation of the yolk stalk ${ }^{22}$. This process remodels the embryo from a flat sheet to a threedimensional tube (Fig. 6). Body wall closure "zips" together the left and right lateral edges of embryo along the ventral midline. This process occurs in both anterior and posterior directions between the anus and the pharyn $\mathrm{x}^{22}$. Incomplete closure of the body wall results in congenital defects such as omphalocele, gastroschisis and exstrophyepispadias complex $(\mathrm{EEC})^{22,23}$. It has been suggested this process requires proliferation and lateral migration of the mesodermal and ectodermal layers of the lateral plate, which causes the body folds to extend and meet at the ventral midline ${ }^{22-27}$. The lateral folding and circumferential (dorsal to ventral) migration of the three germ layers (ectoderm, mesoderm and endoderm) is associated with closure of the gut and urogenital sinuses and formation of the cloacal membrane posteriorly.

Our finding that the genital tubercle arises from left and right genital progenitor fields, which are brought together during body 
wall closure, provides a novel explanation for the association between epispadias and extrophy. Epispadias is an ectopic dorsal opening of the urethra (in contrast to the more common hypospadias, in which the urethra opens ectopically on the ventral side of the penis $)^{28}$. Epispadias can be manifest as a dorsal clefting of the penis or bifurcation of the clitoris, and is often associated with bladder exstrophy, in which the bladder protrudes through the ventral body wall. Our discovery that the phallus has a composite origin, in which the left and right sides are lineage-restricted compartments of progenitor cells that are brought together during body wall closure, suggests that a common mechanism involving disruption of posterior body wall closure could underlie both epispadias and exstrophy, and this might explain their frequent association in humans.

\section{Methods}

Embryos. All experiments were performed in accordance with institutional guidelines and regulations. Fertilized chicken eggs were obtained from Charles River Laboratories and were stored at $10^{\circ} \mathrm{C}$ for up to two weeks before incubation. Eggs were incubated in a humidified incubator at $38^{\circ} \mathrm{C}$. Chick embryos were injected, collected, and fixed prior to hatching. Stages of embryonic development were determined using the Hamburger-Hamilton staging series $^{29}$

Fluorescent Label Application. Fluorescent lipophilic dyes DiI (1,1'-dioctadecyl$3,3,3^{\prime}, 3^{\prime}$-tetramethylindocarbocyanine perchlorate, Molecular probes D-282) and $\mathrm{DiA}$ (4-(4-(dihexadecylamino)styryl)-N-methylpyridinium iodide, Molecular probes D-3883) were used at a stock concentration of $2.5 \mathrm{mg} / \mathrm{mL}$ in dimethylformamide (DMF). Glass capillary needles (FHC Cat. 30-30-0) with an O.D of $1.0 \mathrm{~mm}$ were pulled using a Narishige needle puller to create tapered ends. Capillaries were loaded with dye, mounted in a capillary holder and moved into position for injection using a Narishige micromanipulator mounted to a stereomicroscope. Labeling was performed with a 3-millisecond $(\mathrm{msec})$ pulse from a PicoSpritzer model II (General Valve). Labeling was confirmed by fluorescent microscopy. After 48 hours of incubation at $38^{\circ} \mathrm{C}$, embryos were removed from the egg, dissected in phosphatebuffered saline (PBS), and fixed in $4 \%$ paraformaldehyde (PFA) before imaging and/ or tissue processing.

Histology. After fixation, lower halves of the bodies were dissected and processed through $15 \%$ sucrose/PBS, $30 \%$ sucrose/PBS, and finally $100 \%$ OCT before mounting and freezing on dry ice. Transverse sections were cut at $16 \mu \mathrm{m}$ using a Leica CM3050 $\mathrm{S}$ cryostat with a chamber temperature of $-25^{\circ} \mathrm{C}$.

1. Paulozzi, L. J., Erickson, J. D. \& Jackson, R. J. Hypospadias trends in two US surveillance systems. Pediatrics 100, 831-834 (1997).

2. Paulozzi, L. J. International trends in rates of hypospadias and cryptorchidism. Environ. Health Perspect. 107, 297-302 (1999).

3. Nordenvall, A. S., Frisen, L., Nordenstrom, A., Lichtenstein, P. \& Nordenskjold, A. Population based nationwide study of hypospadias in Sweden, 1973 to 2009 : incidence and risk factors. J. Urol. 191, 783-789 (2014).

4. Gredler, M. L. et al. Evolution of External Genitalia: Insights from Reptilian Development. Sex. Dev. 10.1159/000365771, 311-326 (2014).

5. Leal, F. \& Cohn, M. J. Development of Hemipenes in the Ball Python Snake Python regius. Sex. Dev. 10.1159/000363758 (2014)

6. Larkins, C. E. \& Cohn, M. J. Phallus Development in the Turtle Trachemys scripta. Sex. Dev. 10.1159/000363631 (2014).

7. Herrera, A. M., Brennan, P. L. \& Cohn, M. J. Development of Avian External Genitalia: Interspecific Differences and Sexual Differentiation of the Male and Female Phallus. Sex. Dev. 10.1159/000364927 (2014).

8. Gredler, M. L., Seifert, A. W. \& Cohn, M. J. Morphogenesis and Patterning of the Phallus and Cloaca in the American Alligator, Alligator mississippiensis. Sex. Dev. 10.1159/000364817 (2014).

9. Gredler, M. L., Sanger, T. J. \& Cohn, M. J. Development of the Cloaca, Hemipenes, and Hemiclitores in the Green Anole, Anolis carolinensis. Sex. Dev. 10.1159/ 000363757 (2014).

10. Herrera, A. M., Shuster, S. G., Perriton, C. L. \& Cohn, M. J. Developmental basis of phallus reduction during bird evolution. Curr. Biol. 23, 1065-1074 (2013).

11. Perriton, C. L., Powles, N., Chiang, C., Maconochie, M. K. \& Cohn, M. J. Sonic hedgehog signaling from the urethral epithelium controls external genital development. Dev. Biol. 247, 26-46 (2002).

12. Haraguchi, R. et al. Molecular analysis of coordinated bladder and urogenital organ formation by Hedgehog signaling. Development 134, 525-533 (2007).
13. Seifert, A. W., Harfe, B. D. \& Cohn, M. J. Cell lineage analysis demonstrates an endodermal origin of the distal urethra and perineum. Dev. Biol. 318, 143-152 (2008).

14. Hornbruch, A. \& Wolpert, L. The spatial and temporal distribution of polarizing activity in the flank of the pre-limb-bud stages in the chick embryo. Development 111, 725-731 (1991).

15. Arques, C. G., Doohan, R., Sharpe, J. \& Torres, M. Cell tracing reveals a dorsoventral lineage restriction plane in the mouse limb bud mesenchyme. Development 134, 3713-3722 (2007).

16. Altabef, M., Clarke, J. D. \& Tickle, C. Dorso-ventral ectodermal compartments and origin of apical ectodermal ridge in developing chick limb. Development 124, 4547-4556 (1997).

17. Kimmel, R. A. et al. Two lineage boundaries coordinate vertebrate apical ectodermal ridge formation. Genes Dev. 14, 1377-1389 (2000).

18. Tschopp, P. et al. Nature advance online publication, 5th November 2014 (DOI 10.1038/nature13819).

19. Garcia-Bellido, A., Ripoll, P. \& Morata, G. Developmental compartmentalisation of the wing disk of Drosophila. Nat. New Biol. 245, 251-253 (1973).

20. Huang, R., Zhi, Q., Patel, K., Wilting, J. \& Christ, B. Dual origin and segmental organisation of the avian scapula. Development 127, 3789-3794 (2000)

21. Spitz, F., Gonzalez, F. \& Duboule, D. A global control region defines a chromosomal regulatory landscape containing the HoxD cluster. Cell 113, 405-417 (2003).

22. Sadler, T. W. \& Feldkamp, M. L. The embryology of body wall closure: relevance to gastroschisis and other ventral body wall defects. American journal of medical genetics. Part C, Seminars in Medical Genetics 148C, 180-185 (2008).

23. Sadler, T. W. The embryologic origin of ventral body wall defects. Seminars in Pediatric Surgery 19, 209-214 (2010).

24. Christison-Lagay, E. R., Kelleher, C. M. \& Langer, J. C. Neonatal abdominal wall defects. Seminars in Fetal \& Neonatal Medicine 16, 164-172 (2011).

25. Kulkarni, B. \& Chaudhari, N. Embryogenesis of bladder exstrophy : A new hypothesis. J. Indian Assoc. Pediatr. Surg. 13, 57-60 (2008).

26. Männer, J. \& Kluth, D. The morphogenesis of the exstrophy-epispadias complex: a new concept based on observations made in early embryonic cases of cloacal exstrophy. Anatomy and Embryology 210, 51-57 (2005).

27. Matsumaru, D. et al. Genetic analysis of Hedgehog signaling in ventral body wall development and the onset of omphalocele formation. PloS One 6, e16260, 10.1371/journal.pone.0016260 (2011).

28. Stephens, F. D. \& Hutson, J. M. Differences in embryogenesis of epispadias, exstrophy-epispadias complex and hypospadias. J. Pediatr. Urol. 1, 283-288 (2005).

29. Hamburger, V. \& Hamilton, H. L. A Series of Normal Stages in the Development of the Chick Embryo. Journal of Morphology 88, 49-92 (1951).

\section{Acknowledgments}

We thank M. Maden, B. Harfe, R. Kimball, L. Zhou, and members of our laboratory for critical comments on earlier versions of this manuscript, M. Gredler and O. Tarazona for preparation of schematic diagrams, and P. Tschopp, J. Gros and C. Tabin for stimulating discussions and exchange of data prior to publication. This publication has been funded in part with funds from the National Library of Medicine, National Institutes of Health, under Contract \# HHSN-316-2012-00028-WHHSN-316-2012-00028-W. This study was supported by National Institute of Diabetes and Digestive and Kidney Diseases (U01-DK094523 to M.J.C.) and the Howard Hughes Medical Institute.

\section{Author contributions}

The authors have made the following declarations about their contributions: Conceived the study: M.J.C. Designed the experiments: M.J.C. and A.M.H. Performed the experiments: A.M.H. Analysed the data: M.J.C. and A.M.H. Wrote, edited and approved the manuscript: M.J.C. and A.M.H

\section{Additional information}

Competing financial interests: The authors declare no competing financial interests.

How to cite this article: Herrera, A.M. \& Cohn, M.J. Embryonic origin and compartmental organization of the external genitalia. Sci. Rep. 4, 6896; DOI:10.1038/srep06896 (2014)

This work is licensed under a Creative Commons Attribution-NonCommercialNoDerivs 4.0 International License. The images or other third party material in this article are included in the article's Creative Commons license, unless indicated otherwise in the credit line; if the material is not included under the Creative Commons license, users will need to obtain permission from the license holder in order to reproduce the material. To view a copy of this license, visit http:// creativecommons.org/licenses/by-nc-nd/4.0/ 